\title{
On Delayed Genetic Regulatory Networks With Polytopic Uncertainties: Robust Stability Analysis
}

\author{
Zidong Wang*, Huijun Gao, Jinde Cao, and Xiaohui Liu
}

\begin{abstract}
In this paper, we investigate the robust asymptotic stability problem of genetic regulatory networks with time-varying delays and polytopic parameter uncertainties. Both cases of differentiable and nondifferentiable time-delays are considered, and the convex polytopic description is utilized to characterize the genetic network model uncertainties. By using a Lyapunov functional approach and linear matrix inequality (LMI) techniques, the stability criteria for the uncertain delayed genetic networks are established in the form of LMIs, which can be readily verified by using standard numerical software. An important feature of the results reported here is that all the stability conditions are dependent on the upper and lower bounds of the delays, which is made possible by using up-to-date techniques for achieving delay dependence. Another feature of the results lies in that a novel Lyapunov functional dependent on the uncertain parameters is utilized, which renders the results to be potentially less conservative than those obtained via a fixed Lyapunov functional for the entire uncertainty domain. A genetic network example is employed to illustrate the applicability and usefulness of the developed theoretical results.
\end{abstract}

Index Terms-Genetic regulatory network, linear matrix inequality, robust stability, time-varying delay.

\section{INTRODUCTION}

$\mathbf{T}$ HE PAST decade has seen significant progress in genome sequencing and gene recognition, which gives rise to a large volume of experimental data [10], [29]. However, very few breakthroughs have been made on how to utilize these data to understand gene functions (such as the mechanism that genes and proteins interact with one another to form systems or networks that perform sophisticated and complicated biological functions). Recent study on genetic networks with mathematical tools is an important attempt towards this challenging problem, aiming at filling the gap between the genome sequencing and

\footnotetext{
Manuscript received February 5, 2007; revised July 23, 2007. This work was supported in part by the Biotechnology and Biological Sciences Research Council (BBSRC) of the U.K. under Grants BB/C506264/1 and 100/EGM17735, in part by the Engineering and Physical Sciences Research Council (EPSRC) of the U.K. under Grants GR/S27658/01 and EP/C524586/1, in part by an International Joint Project sponsored by the Royal Society of the U.K., in part by the Nuffield Foundation of the U.K. under Grant NAL/00630/G, and in part by the Alexander von Humboldt Foundation of Germany. Asterisk indicates corresponding author.

*Z. Wang is with the Department of Information Systems and Computing, Brunel University, Uxbridge, Middlesex UB8 3PH, U.K. (e-mail: Zidong.Wang@brunel.ac.uk).

$\mathrm{X}$. Liu is with the Department of Information Systems and Computing, Brunel University, Uxbridge, Middlesex UB8 3PH, U.K.

$\mathrm{H}$. Gao is with the Space Control and Inertial Technology Research Center, Harbin Institute of Technology, Harbin 150001, China.

J. Cao is with the Department of Mathematics, Southeast University, Nanjing 210096, China.

Color versions of one or more of the figures in this paper are available online at http://ieeexplore.ieee.org.

Digital Object Identifier 10.1109/TNB.2008.2000746
}

the understanding of gene functions. Theoretical studies on genetic networks may not only contribute to the understanding of the gene functions, but also have potential significance on engineering applications, such as developing circuits and systems with biotechnological design principles of synthetic genetic regulatory networks and new kinds of integrated circuits like neurochips learnt from biological neural networks [9], [15].

By genetic networks, we refer to biochemically dynamic systems, metabolic dynamic systems, as well as signal transduction dynamic systems. For example, for biochemically dynamic systems, the nodes indicate the biochemicals, and the couplings represent the biochemical interactions. This kind of networks have become a powerful tool for studying gene regulation processes in living organisms. Generally speaking, so far, two types of genetic network models have been proposed and utilized [3], [7], [8], [21], [22], [31]: Boolean model (discrete model) and differential equation model (continuous model). In the discrete model, only two states (that is, ON and OFF) are used to express the activity of each gene, and the state of a gene is determined by a Boolean function of the states of other related genes. In the continuous model, usually a differential equation is utilized to describe the whole network, with the state variables describing the concentrations of gene products, such as mRNAs and proteins. Using continuous values, the second approach is considered more accurate, and being able to provide more detailed understanding and insights of the dynamic behavior exhibited by biological systems. A combination of these two models has led to the so-called hybrid model, which describes genetic networks in both switching and smoothly varying manners [6], [26].

Recent studies on genetic regulatory networks are fruitful, and many important results have been reported in the literature. These results make significant contributions for discovering higher order structure of an organism and for gaining deep insights into both static and dynamic behaviors of genetic networks by extracting functional information from observation data. Similarly to other dynamic systems, stability is a key property for genetic regulatory networks, and readers are referred to [1], [2], [7], [22], [32] for some recent results. It has been recognized that the slow processes of transcription, translation, and translocation or the finite switching speed of amplifiers will inevitable cause time delays, which should be taken into account in the biological systems or artificial genetic networks in order to have more accurate models [24]. It has been shown in [27], by mathematically modelling recent data, that the observed oscillatory expression and activity of three proteins is most likely to be driven by transcriptional delays, and delays can have significant impact both on the dynamical behavior of models and on numerical parameter prediction. Therefore, the effects 
of transcriptional delays should be assessed in the dynamics of genetic networks whose time scales are short and transcription is regulated by feedback. However, the incorporation of time delays brings great challenges, as the dynamics of the genetic networks will generally change from finite dimension to infinite dimension.

Theoretical results obtained for genetic networks with or without time delays are scattered in the literature. To mention just a few, the regulatory effect on the RNA polymerase binding of the transcription factor proteins produced by other genes has been analyzed in [16], where a dynamic system has been modeled for such an interacting chain and its behavior has been analyzed under various assumptions concerning the dependence of binding on the transcription factor concentration. In [1], a simple gene circuit has been constructed, which consists of regulator and transcriptional repressor modules in Escherichia coli, and the gain of stability produced by negative feedback has been given. A model has been presented in [7] for genetic regulatory networks with time delays and the nonlinear properties of the model have been analyzed in terms of local stability and bifurcation. In [22], a nonlinear model for genetic regulatory networks with SUM regulatory functions has been presented, and the stabilities of genetic networks with time-varying delays or stochastic perturbations have been investigated. Very recently, in [30], the asymptotic stability has been discussed for the delayed GRNs with SUM regulatory logic, where time delays are assumed to be time-varying and belong to given intervals.

On the other hand, from the systems point of view, when modeling a dynamic system, one can hardly obtain an exact model. In other words, there is always some error between the mathematical model and the physical system, which can be represented in the form of external perturbations, parameter fluctuations and unstructured dynamics [38]. This also applies to the modelling of genetic regulatory networks, and the analysis results without taking into account modeling uncertainties may not be as useful as expected in real applications. Therefore, it is essential and important to investigate the robust stability of the genetic regulatory network with parameter uncertainties/perturbations. To the best of the authors' knowledge, up to now, little effort has been made towards this challenging problem, which motivates the present study.

In this paper, we are concerned with the robust asymptotic stability of genetic regulatory networks with time-varying delays and parameter uncertainties. Two cases of time delays are considered (differentiable and nondifferentiable), and the parameter uncertainty considered here is assumed to be of polytopic-type. By using a Lyapunov functional approach and the linear matrix inequality (LMI) techniques, robust asymptotic stability is established in terms of LMIs that can be readily solved by using standard numerical software (such as Matlab) [5], [11]. An important feature of the results reported here is that all the conditions are dependent on the upper and lower bounds of the delays, and the traditional assumption that the derivatives of the delays are less than 1 (as used in [22]) is no longer required in our analysis. Another feature of the results lies in that an advanced Lyapunov functional dependent on the uncertain parameters has been utilized, which renders the results to be potentially less conservative than those obtained by using a fixed Lyapunov functional for the entire uncertainty domain.
Notations: The notations used throughout the paper are fairly standard. The superscript " $T$ " stands for matrix transposition; $\mathbb{R}^{n}$ denotes the $n$-dimensional Euclidean space (with $\mathbb{R}$ used for the case $n=1$ ); $P>0$ means that the matrix $P$ is real, symmetric, and positive definite; $I$ and 0 represent identity matrix and zero matrix, respectively. For symmetric block matrices or long matrix expressions, an asterisk $(*)$ is used to represent a term that is induced by symmetry. Matrices, if their dimensions are not explicitly stated, are assumed to be compatible for algebraic operations.

\section{Model AND PRELIMINARIES}

To facilitate the readers, before the problem formulation, we introduce the commonly used gene network models in this section. Let us start from a single gene auto-regulatory genetic network [36]

$$
\begin{aligned}
\dot{\phi}(t) & =K[\psi(t)]-\kappa \phi(t), \\
\dot{\psi}(t) & =v \phi(t)-\theta \psi(t) .
\end{aligned}
$$

Here, $\phi(t) \in \mathbb{R}$ and $\psi(t) \in \mathbb{R}$ are the concentrations of mRNA and protein, and their rates of degradation are denoted by, respectively, $\kappa$ and $\theta . v$ is the translation rate, and the function $K[\psi(t)]$ represents the feedback regulation of the protein on transcription. Taking into account the transcriptional time delay, we have the following model [27]:

$$
\begin{aligned}
\dot{\phi}(t) & =K[\psi(t-d(t))]-\kappa \phi(t), \\
\dot{\psi}(t) & =v \phi(t)-\theta \psi(t) .
\end{aligned}
$$

It has been shown in [27] that the observed oscillatory expression and activity of three proteins are most likely to be driven by transcriptional delays.

In a genetic regulatory network, a number of genes interact and regulate the expression of other genes by proteins, the gene derivatives. The change in expression of a gene is governed by the stimulation and inhibition of proteins in transcriptional, translational, and post-translational processes. The following differential equations have been used recently to describe the genetic regulatory networks [7], [9]:

$$
\begin{aligned}
\dot{\phi}_{i}(t)=- & \kappa_{i} \phi_{i}(t)+K_{i}\left(\psi_{1}(t-\tau(t)), \psi_{2}(t-\tau(t)),\right. \\
& \left.\ldots, \psi_{n}(t-\tau(t))\right), \\
\dot{\psi}_{i}(t)=- & \theta_{i} \psi_{i}(t)+v_{i} \phi_{i}(t-d(t)), \quad i=1,2, \ldots, n,
\end{aligned}
$$

where $\phi_{i}(t) \in \mathbb{R}$ and $\psi_{i}(t) \in \mathbb{R}$ are the concentrations of mRNA and protein of the $i$ th node, whose decay rates are denoted by $\kappa_{i}$ and $\theta_{i}$ respectively; $v_{i}$ is the translation rate, and the function $K_{i}$ represents the feedback regulation of the protein on the transcription, which is generally a nonlinear function but has a form of monotonicity with each variable [8], [31]. From (3) we can see that, in this network, for any single gene $i$, there is only one output $\psi_{i}(t-\tau(t))$ to other genes, but multiple inputs $\psi_{j}(t-$ $\tau(t)), j=1,2, \ldots, n$ from other genes. Readers are referred to [7] for more detailed information about the structure and regulation mechanism of the genetic network. Being a monotonic increasing or decreasing regulatory function, $K_{i}$ usually takes the Michaelis-Menten or Hill form. In this paper, the function $K_{i}$ is taken as $K_{i}\left(\psi_{1}(t), \psi_{2}(t), \ldots, \psi_{n}(t)\right)=\sum_{j=1}^{n} K_{i j}\left(\psi_{j}(t)\right)$, which is called SUM logic [20], [37] because each transcription 
factor acts additively to regulate the $i$ th gene. $K_{i j}$ is a monotonic function of the Hill form, that is, if transcription factor $j$ is an activator of gene $i$, then

$$
K_{i j}\left(\psi_{j}(t)\right)=\rho_{i j} \frac{\left(\frac{\psi_{j}(t)}{\beta_{j}}\right)^{H_{j}}}{1+\left(\frac{\psi_{j}(t)}{\beta_{j}}\right)^{H_{j}}}
$$

and if transcription factor $j$ is a repressor of gene $i$, then

$$
\begin{aligned}
K_{i j}\left(\psi_{j}(t)\right) & =\rho_{i j} \frac{1}{1+\left(\frac{\psi_{j}(t)}{\beta_{j}}\right)^{H_{j}}} \\
& =\rho_{i j}\left(1-\frac{\left(\frac{\psi_{j}(t)}{\beta_{j}}\right)^{H_{j}}}{1+\left(\frac{\psi_{j}(t)}{\beta_{j}}\right)^{H_{j}}}\right) .
\end{aligned}
$$

Here, $H_{j}$ is the Hill coefficients; $\beta_{j}$ is a positive constant; $\rho_{i j}$ is a bounded constant, which is the dimensionless transcriptional rate of transcription factor $j$ to $i$. Based on (4) and (5), the gene networks in (3) can be rewritten as

$$
\begin{aligned}
\dot{\phi}_{i}(t) & =-\kappa_{i} \phi_{i}(t)+\sum_{j=1}^{n} b_{i j} f_{j}\left(\psi_{j}(t-\tau(t))\right)+\rho_{i}, \\
\dot{\psi}_{i}(t) & =-\theta_{i} \psi_{i}(t)+v_{i} \phi_{i}(t-d(t)), \\
f_{j} & \triangleq \frac{\left(\frac{\psi_{j}(t)}{\beta_{j}}\right)^{H_{j}}}{1+\left(\frac{\psi_{j}(t)}{\beta_{j}}\right)^{H_{j}}}, \\
\rho_{i} & =\sum_{j \in \mathcal{I}_{i}} \rho_{i j}
\end{aligned}
$$

where $\mathcal{I}_{i}$ is the set of all the $j$ which is a repressor of gene $i$, and $B=\left[b_{i j}\right] \in \mathbb{R}^{n \times n}$ is defined as follows: $b_{i j}=\rho_{i j}$ if transcription factor $j$ is an activator of gene $i ; b_{i j}=0$ if there is no link from gene $j$ to gene $i$; and $b_{i j}=-\rho_{i j}$ if transcription factor $j$ is a repressor of gene $i$. By denoting

$$
\begin{aligned}
\bar{\phi}(t) & =\left[\begin{array}{c}
\phi_{1}(t) \\
\phi_{2}(t) \\
\vdots \\
\phi_{n}(t)
\end{array}\right], \quad \bar{\psi}(t)=\left[\begin{array}{c}
\psi_{1}(t) \\
\psi_{2}(t) \\
\vdots \\
\psi_{n}(t)
\end{array}\right], \\
\rho & \left.=\left[\begin{array}{c}
\rho_{1} \\
\rho_{2} \\
\vdots \\
\rho_{n}
\end{array}\right], \quad \bar{\phi}(t-d(t))\right)=\left[\begin{array}{c}
\phi_{1}(t-d(t)) \\
\phi_{2}(t-d(t)) \\
\vdots \\
\phi_{n}(t-d(t))
\end{array}\right], \\
f(\bar{\psi}(t-\tau(t)))= & {\left[\begin{array}{c}
f_{1}\left(\psi_{1}(t-\tau(t))\right) \\
f_{2}\left(\psi_{2}(t-\tau(t))\right) \\
\vdots \\
f_{n}\left(\psi_{n}(t-\tau(t))\right)
\end{array}\right], } \\
A= & \operatorname{diag}\left\{-\kappa_{1},-\kappa_{2}, \ldots,-\kappa_{n}\right\}, \\
C= & \operatorname{diag}\left\{-\theta_{1},-\theta_{2}, \ldots,-\theta_{n}\right\}, \\
D & =\operatorname{diag}\left\{v_{1}, v_{2}, \ldots, v_{n}\right\}
\end{aligned}
$$

system (6) can be rewritten into the following compact matrix form:

$$
\begin{aligned}
\bar{\phi}(t) & =A \bar{\phi}(t)+B f(\bar{\psi}(t-\tau(t)))+\rho, \\
\bar{\psi}(t) & =C \bar{\psi}(t)+D \bar{\phi}(t-d(t)) .
\end{aligned}
$$

Let $\bar{\phi}^{*} \triangleq\left[\begin{array}{llll}\phi_{1}^{*} & \phi_{2}^{*} & \cdots & \phi_{n}^{*}\end{array}\right]^{T}$ and $\bar{\psi}^{*} \triangleq$ $\left[\begin{array}{llll}\psi_{1}^{*} & \psi_{2}^{*} & \cdots & \psi_{n}^{*}\end{array}\right]^{T}$ be an equilibrium of (7). That is, $\left(\bar{\phi}^{*}, \bar{\psi}^{*}\right)$ is the solution of equation

$$
\begin{aligned}
& 0=A \bar{\phi}^{*}+B f\left(\bar{\psi}^{*}\right)+\rho, \\
& 0=C \bar{\psi}^{*}+D \bar{\phi}^{*} .
\end{aligned}
$$

By using the following transformation:

$$
\Phi(t)=\bar{\phi}(t)-\bar{\phi}^{*}, \quad \Psi(t)=\bar{\psi}(t)-\bar{\psi}^{*}
$$

an intended equilibrium point $\left(\bar{\phi}^{*}, \bar{\psi}^{*}\right)$ of system (7) can be shifted to the origin

$$
\begin{aligned}
& \dot{\Phi}(t)=A \Phi(t)+B h(\Psi(t-\tau(t))) \\
& \dot{\Psi}(t)=C \Psi(t)+D \Phi(t-d(t))
\end{aligned}
$$

where

$$
\begin{aligned}
& \Phi(t)=\left[\begin{array}{c}
\Phi_{1}(t) \\
\Phi_{2}(t) \\
\vdots \\
\Phi_{n}(t)
\end{array}\right], \quad \Psi(t)=\left[\begin{array}{c}
\Psi_{1}(t) \\
\Psi_{2}(t) \\
\vdots \\
\Psi_{n}(t)
\end{array}\right], \\
& h(\Psi(t))= {\left[\begin{array}{c}
h_{1}\left(\Psi_{1}(t)\right) \\
h_{2}\left(\Psi_{2}(t)\right. \\
\vdots \\
h_{n}\left(\Psi_{n}(t)\right)
\end{array}\right], } \\
& \text { and } \\
& h_{i}\left(\Psi_{i}(t)\right)=f_{i}\left(\bar{\phi}_{i}(t)+\bar{\phi}_{i}^{*}\right)-f_{i}\left(\bar{\phi}_{i}^{*}\right) .
\end{aligned}
$$

Since $f_{i}$ is a monotonically increasing function with saturation, it satisfies the following constraint:

$$
0 \leq \frac{f_{i}(x)-f_{i}(y)}{x-y} \leq a_{i}
$$

for all $x \neq y \in \mathbb{R}$. Thus, the function $h_{i}$ satisfies the sector condition

$$
0 \leq \frac{h_{i}(x)}{x} \leq a_{i}
$$

which is equivalent to the following one:

$$
h_{i}(x)\left(h_{i}(x)-a_{i} x\right) \leq 0 .
$$

It can be noticed that the genetic network in (3) has now been transformed into a kind of Lur'e system. In general, in modeling a gene regulatory network, one can hardly obtain exact values of the matrices in (9). Therefore, we consider the following uncertain genetic networks with time-varying delays:

$$
\begin{aligned}
\dot{\Phi}(t) & =A_{\lambda} \Phi(t)+B_{\lambda} h(\Psi(t-\tau(t))), \\
\dot{\Psi}(t) & =C_{\lambda} \Psi(t)+D_{\lambda} \Phi(t-d(t)),
\end{aligned}
$$

where $A_{\lambda}, W_{\lambda}, C_{\lambda}, D_{\lambda}$ represent uncertain system matrices. It is assumed that

$$
\Omega_{\lambda} \triangleq\left(A_{\lambda}, B_{\lambda}, C_{\lambda}, D_{\lambda}\right) \in \Gamma
$$

where $\Gamma$ is a given convex bounded polyhedral domain described by $s$ vertices

$$
\Gamma \triangleq\left\{\Omega_{\lambda} \mid \Omega_{\lambda}=\sum_{i=1}^{s} \lambda_{i} \Omega_{i} ; \quad \sum_{i=1}^{s} \lambda_{i}=1, \lambda_{i} \geq 0\right\}
$$

and $\Omega_{i} \triangleq\left(A_{i}, B_{i}, C_{i}, D_{i}\right)$ denotes the $i$ th vertex of the polytope. 
Remark 1: The parameter uncertainties considered in this paper are assumed to be of polytopic type, entering into all the matrices of the genetic network model. The polytopic uncertainty has been widely used in the problems of robust analysis of uncertain dynamic systems (see, for instance, [13] and the references therein), and many practical systems possess parameter uncertainties which can be either exactly modelled or overbounded by the polytopic uncertainty $\Gamma$.

Remark 2: Note that system (11) involves the uncertain parameter $\lambda$ that forms the polytopic uncertainty $\Gamma$. It is clear from the sector condition on the function $h_{i}$ that the equilibrium (if it exists) of (11) is just the trivial (zero) solution. Obviously, the existence of the equilibrium of (11) is dependent on the uncertain parameter $\lambda$. Therefore, this paper aims to deal with the analysis problem for the robust stability of (11), that is, how to establish sufficient conditions under which the system (11) remains asymptotically stable for all admissible parameter uncertainties in $\Gamma$ and make sure that such conditions are as less conservative as possible. Our next goal, which is one of our future research topics, is then to deal with the synthesis problem, i.e., how to "stabilize" a possibly unstable gene regulatory network by developing control (or intervention) strategies to modify dynamical behavior. It should be pointed out that, when the gene network is viewed as a dynamical system, external control (or intervention) can be applied to make the controlled network achieve desired behaviors such as the stability of the gene expression level regardless of the parameter variation within a certain range.

Remark 3: Time delays are frequently encountered in not only the biological networks but also many other practical engineering systems, such as communication, electronics, hydraulic, and chemical systems. It is now well known that time delay is one of the main causes of instability and poor performance of a control system [25]. In the past decade, stability analysis and synthesis problems for various time-delay systems have gained considerable research interests and a large amount of results have appeared in the literature, see, e.g., [4], [12], [13], [17], [22], [33], and [35]. Recently, the problem of delay-dependent stability analysis for time-delay systems has received much attention and fruitful results have been reported. For example, some delay-dependent stability conditions for continuous-time systems with constant time-delays have been proposed in [28]. For the case when time-varying delays are considered, corresponding results can be found in [14], [19], [23]. It is noticed that, up to now, most existing results are restrictive to some extent since they have assumed that either the derivatives of the delays are less than 1 (e.g., [22]) or the employed Lyapunov functional is fixed for the entire uncertainty domain [33]-[35]. Therefore, one of the objectives in this paper is to remove the restrictions and reduce the possible conservatism by introducing an advanced parameter-dependent Lyapunov functional.

In this paper, we assume that the time-varying delays $d(t)$ and $\tau(t)$ are continuous. Now, let us consider the following two cases of the time-varying delays:

$$
\begin{aligned}
& \text { Case I } \quad d_{m} \leq d(t) \leq d_{M}<\infty, \quad \dot{d}(t) \leq \varepsilon_{d}<\infty \\
& \quad \tau_{m} \leq \tau(t) \leq \tau_{M}<\infty, \quad \dot{\tau}(t) \leq \varepsilon_{\tau}<\infty \\
& \text { Case II } \quad d_{m} \leq d(t) \leq d_{M}<\infty \\
& \quad \tau_{m} \leq \tau(t) \leq \tau_{M}<\infty
\end{aligned}
$$

Remark 4: The difference between the above two delay cases lies in whether the time delays are differentiable. It is worth noting that in either case, different from [22], we do not require the derivatives of the delays to be less than 1 . We will give stability conditions for the above two cases in the next section.

\section{Robust Stability of Genetic Networks}

In this section, we present robust stability conditions for the genetic networks with time-varying delays described in the above section. Firstly we consider Case I.

Theorem 1: The genetic network in (11) with time varying delays satisfying Case I is robustly asymptotically stable if there exist matrices $P_{i} \triangleq\left[\begin{array}{ll}P_{1 i} & P_{2 i} \\ P_{2 i}^{T} & P_{3 i}\end{array}\right]>0, Q_{j i} \geq 0, Z_{j i}>0$, $i=1, \ldots, s, j=1,2,3,4, X, Y$ and diagonal matrices $R_{i}$, $i=1, \ldots, s$, satisfying

$$
\Xi_{i}+\Pi_{i}+\Pi_{i}^{T}<0, \quad i=1, \ldots, s
$$

where

$$
\Xi_{i}=\left[\begin{array}{ll}
\Sigma_{11 i} & \Sigma_{12 i} \\
\Sigma_{12 i}^{T} & \Sigma_{22 i}
\end{array}\right]
$$

with

$$
\begin{aligned}
& \Sigma_{11 i}=\left[\begin{array}{ccccc}
\Xi_{1 i} & \frac{1}{d_{m}} Z_{1 i} & 0 & P_{1 i} & 0 \\
* & \Xi_{2 i} & \frac{1}{d_{M}-d_{m}} Z_{2 i} & 0 & 0 \\
* & * & \Xi_{3 i} & 0 & 0 \\
* & * & * & \Xi_{4 i} & P_{2 i} \\
* & * & * & * & \Xi_{5 i}
\end{array}\right], \\
& \Sigma_{12 i}=\left[\begin{array}{cccc}
0 & 0 & 0 & P_{2 i} \\
0 & 0 & 0 & 0 \\
0 & 0 & 0 & 0 \\
0 & 0 & 0 & 0 \\
\frac{1}{\tau_{m}} Z_{3 i} & 0 & 0 & P_{3 i}
\end{array}\right], \\
& \Sigma_{22 i}=\left[\begin{array}{cccc}
\Xi_{6 i} & \frac{1}{\tau_{M}-\tau_{m}} Z_{4 i} & 0 & 0 \\
* & \Xi_{7 i} & \Lambda R_{i} & 0 \\
* & * & -2 R_{i} & 0 \\
* & * & * & \Xi_{8 i}
\end{array}\right] \text {, } \\
& \Xi_{1 i}=Q_{1 i}-\frac{1}{d_{m}} Z_{1 i}, \\
& \Xi_{2 i}=-\left(Q_{1 i}-Q_{2 i}\right)-\frac{1}{d_{m}} Z_{1 i}-\frac{1}{d_{M}-d_{m}} Z_{2 i}, \\
& \Xi_{3 i}=-\left(1-\varepsilon_{d}\right) Q_{2 i}-\frac{1}{d_{M}-d_{m}} Z_{2 i} \text {, } \\
& \Xi_{4 i}=d_{m} Z_{1 i}+\left(d_{M}-d_{m}\right) Z_{2 i} \text {, } \\
& \Xi_{5 i}=Q_{3 i}-\frac{1}{\tau_{m}} Z_{3 i} \text {, } \\
& \Xi_{6 i}=-\left(Q_{3 i}-Q_{4 i}\right)-\frac{1}{\tau_{m}} Z_{3 i}-\frac{1}{\tau_{M}-\tau_{m}} Z_{4 i}, \\
& \Xi_{7 i}=-\left(1-\varepsilon_{\tau}\right) Q_{4 i}-\frac{1}{\tau_{M}-\tau_{m}} Z_{4 i}, \\
& \Xi_{8 i}=\tau_{m} Z_{3 i}+\left(\tau_{M}-\tau_{m}\right) Z_{4 i} \text {, } \\
& \Pi_{i}=X\left[\begin{array}{lllllllll}
A_{i} & 0 & 0 & -I & 0 & 0 & 0 & B_{i} & 0
\end{array}\right] \\
& +Y\left[\begin{array}{lllllllll}
0 & 0 & D_{i} & 0 & C_{i} & 0 & 0 & 0 & -I
\end{array}\right] \text {, } \\
& \Lambda=\operatorname{diag}\left\{a_{1}, a_{2}, \ldots, a_{n}\right\} \text {. }
\end{aligned}
$$


Proof: Consider the following Lyapunov-Krasovskii functional:

$$
V(t)=V_{1}(t)+V_{2}(t)+V_{3}(t)
$$

where

$$
\begin{aligned}
& V_{1}(t)=\left[\begin{array}{c}
\Phi(t) \\
\Psi(t)
\end{array}\right]^{T}\left[\begin{array}{ll}
P_{1 \lambda} & P_{2 \lambda} \\
P_{2 \lambda}^{T} & P_{3 \lambda}
\end{array}\right]\left[\begin{array}{l}
\Phi(t) \\
\Psi(t)
\end{array}\right], \\
& V_{2}(t)=\int_{t-d_{m}}^{t} \Phi^{T}(s) Q_{1 \lambda} \Phi(s) d s \\
& +\int_{t-d(t)}^{t-d_{m}} \Phi^{T}(s) Q_{2 \lambda} \Phi(s) d s \\
& +\int_{t-\tau_{m}}^{t} \Psi^{T}(s) Q_{3 \lambda} \Psi(s) d s \\
& +\int_{t-\tau(t)}^{t-\tau_{m}} \Psi^{T}(s) Q_{4 \lambda} \Psi(s) d s, \\
& V_{3}(t)=\int_{-d_{m}}^{0} \int_{\beta}^{0} \dot{\Phi}^{T}(t+\alpha) Z_{1 \lambda} \dot{\Phi}(t+\alpha) d \alpha d \beta \\
& +\int_{-d_{M}}^{-d_{m}} \int_{\beta}^{0} \dot{\Phi}^{T}(t+\alpha) Z_{2 \lambda} \dot{\Phi}(t+\alpha) d \alpha d \beta \\
& +\int_{-\tau_{m}}^{0} \int_{\beta}^{0} \dot{\Psi}^{T}(t+\alpha) Z_{3 \lambda} \dot{\Psi}(t+\alpha) d \alpha d \beta \\
& +\int_{-\tau_{M}}^{-\tau_{m}} \int_{\beta}^{0} \dot{\Psi}^{T}(t+\alpha) Z_{4 \lambda} \dot{\Psi}(t+\alpha) d \alpha d \beta, \\
& \text { and } \\
& {\left[\begin{array}{ll}
P_{1 \lambda} & P_{2 \lambda} \\
P_{2 \lambda}^{T} & P_{3 \lambda}
\end{array}\right] \triangleq \sum_{i=1}^{s} \lambda_{i}\left[\begin{array}{ll}
P_{1 i} & P_{2 i} \\
P_{2 i}^{T} & P_{3 i}
\end{array}\right]>0,} \\
& Q_{j \lambda} \triangleq \sum_{i=1}^{s} \lambda_{i} Q_{j i} \geq 0 \text {, } \\
& Z_{j \lambda} \triangleq \sum_{i=1}^{s} \lambda_{i} Z_{j i}>0
\end{aligned}
$$

Then, along the solution of (11), the time derivative of $V(t)$ is given by

$$
\begin{aligned}
\dot{V}_{1}(t)= & 2\left[\begin{array}{l}
\Phi(t) \\
\Psi(t)
\end{array}\right]^{T}\left[\begin{array}{cc}
P_{1 \lambda} & P_{2 \lambda} \\
P_{2 \lambda}^{T} & P_{3 \lambda}
\end{array}\right]\left[\begin{array}{c}
\dot{\Phi}(t) \\
\dot{\Psi}(t)
\end{array}\right] \\
\dot{V}_{2}(t)= & \Phi^{T}(t) Q_{1 \lambda} \Phi(t)-\Phi^{T}\left(t-d_{m}\right) Q_{1 \lambda} \Phi\left(t-d_{m}\right) \\
& +\Phi^{T}\left(t-d_{m}\right) Q_{2 \lambda} \Phi\left(t-d_{m}\right) \\
& -(1-\dot{d}(t)) \Phi^{T}(t-d(t)) Q_{2 \lambda} \Phi(t-d(t)) \\
& +\Psi^{T}(t) Q_{3 \lambda} \Psi(t)-\Psi^{T}\left(t-\tau_{m}\right) Q_{3 \lambda} \Psi\left(t-\tau_{m}\right) \\
& +\Psi^{T}\left(t-\tau_{m}\right) Q_{4 \lambda} \Psi\left(t-\tau_{m}\right) \\
& -(1-\dot{\tau}(t)) \Psi^{T}(t-\tau(t)) Q_{4 \lambda} \Psi(t-\tau(t)) \\
\leq & \Phi^{T}(t) Q_{1 \lambda} \Phi(t) \\
& -\Phi^{T}\left(t-d_{m}\right)\left(Q_{1 \lambda}-Q_{2 \lambda}\right) \Phi\left(t-d_{m}\right) \\
& -\left(1-\varepsilon_{d}\right) \Phi^{T}(t-d(t)) Q_{2 \lambda} \Phi(t-d(t)) \\
& +\Psi^{T}(t) Q_{3 \lambda} \Psi(t) \\
& -\Psi^{T}\left(t-\tau_{m}\right)\left(Q_{3 \lambda}-Q_{4 \lambda}\right) \Psi\left(t-\tau_{m}\right) \\
& -\left(1-\varepsilon_{\tau}\right) \Psi^{T}(t-\tau(t)) Q_{4 \lambda} \Psi(t-\tau(t))
\end{aligned}
$$

$$
\begin{aligned}
\leq & -\frac{1}{\tau_{m}}\left(\int_{t-\tau_{m}}^{t} \dot{\Psi}(\alpha) d \alpha\right)^{T} Z_{3 \lambda}\left(\int_{t-\tau_{m}}^{t} \dot{\Psi}(\alpha) d \alpha\right) \\
=- & \frac{1}{\tau_{m}}\left(\Psi(t)-\Psi\left(t-\tau_{m}\right)\right)^{T} Z_{3 \lambda}\left(\Psi(t)-\Psi\left(t-\tau_{m}\right)\right), \\
& -\int_{t-\tau_{M}}^{t-\tau_{m}} \dot{\Phi}^{T}(\alpha) Z_{4 \lambda} \dot{\Phi}(\alpha) d \alpha \\
\leq & -\int_{t-\tau(t)}^{t-\tau_{m}} \dot{\Psi}^{T}(\alpha) Z_{4 \lambda} \dot{\Psi}(\alpha) d \alpha \\
\leq & -\frac{1}{\tau(t)-\tau_{m}}\left(\int_{t-\tau(t)}^{t-\tau_{m}} \dot{\Psi}(\alpha) d \alpha\right)^{T} \\
& \times Z_{4 \lambda}\left(\int_{t-\tau(t)}^{t-\tau_{m}} \dot{\Psi}(\alpha) d \alpha\right) \\
\leq & -\frac{1}{\tau_{M}-\tau_{m}}\left(\Psi\left(t-\tau_{m}\right)-\Psi(t-\tau(t))\right)^{T} \\
& \times Z_{4 \lambda}\left(\Psi\left(t-\tau_{m}\right)-\Psi(t-\tau(t))\right) .
\end{aligned}
$$


In addition, based on (9), for any matrices $X$ and $Y$, we have

$$
\begin{aligned}
2 \zeta^{T}(t) X\left[A_{\lambda} \Phi(t)+B_{\lambda} h(\Psi(t-\tau(t)))-\dot{\Phi}(t)\right] & =0 \\
2 \zeta^{T}(t) Y\left[C_{\lambda} \Psi(t)+D_{\lambda} \Phi(t-d(t))-\dot{\Psi}(t)\right] & =0
\end{aligned}
$$

where

$$
\zeta(t)=\left[\begin{array}{c}
\Phi(t) \\
\Phi\left(t-d_{m}\right) \\
\Phi(t-d(t)) \\
\dot{\Phi}(t) \\
\Psi(t) \\
\Psi\left(t-\tau_{m}\right) \\
\Psi(t-\tau(t)) \\
h(\Psi(t-\tau(t))) \\
\dot{\Psi}(t)
\end{array}\right] .
$$

Define

$$
R_{\lambda} \triangleq \sum_{i=1}^{s} \lambda_{i} R_{i}=\operatorname{diag}\left\{r_{1 \lambda}, r_{2 \lambda}, \ldots, r_{n \lambda}\right\}
$$

From the sector condition, we have

$$
\begin{aligned}
&-2 \sum_{i=1}^{n} r_{i \lambda} h_{i}\left(\Psi_{i}(t-\tau(t))\right)\left(h_{i}\left(\Psi_{i}(t-\tau(t))\right)\right. \\
&\left.-a_{i} \Psi_{i}(t-\tau(t))\right) \geq 0
\end{aligned}
$$

that is,

$$
\begin{aligned}
& -2 h^{T}(\Psi(t-\tau(t))) R_{\lambda} h(\Psi(t-\tau(t))) \\
& +2 h^{T}(\Psi(t-\tau(t))) R_{\lambda} \Lambda \Psi(t-\tau(t)) \geq 0 .
\end{aligned}
$$

Then, from (15)-(19) we have

$$
\begin{aligned}
\dot{V}(t) \leq & 2\left[\begin{array}{l}
\Phi(t) \\
\Psi(t)
\end{array}\right]^{T}\left[\begin{array}{ll}
P_{1 \lambda} & P_{2 \lambda} \\
P_{2 \lambda}^{T} & P_{3 \lambda}
\end{array}\right]\left[\begin{array}{c}
\dot{\Phi}(t) \\
\dot{\Psi}(t)
\end{array}\right] \\
& +\Phi^{T}(t) Q_{1 \lambda} \Phi(t)-\Phi^{T}\left(t-d_{m}\right) \\
& \times\left(Q_{1 \lambda}-Q_{2 \lambda}\right) \Phi\left(t-d_{m}\right) \\
& -\left(1-\varepsilon_{d}\right) \Phi^{T}(t-d(t)) Q_{2 \lambda} \Phi(t-d(t)) \\
& +\Psi^{T}(t) Q_{3 \lambda} \Psi(t)-\Psi^{T}\left(t-\tau_{m}\right) \\
& \times\left(Q_{3 \lambda}-Q_{4 \lambda}\right) \Psi\left(t-\tau_{m}\right) \\
& -\left(1-\varepsilon_{\tau}\right) \Psi^{T}(t-\tau(t)) Q_{4 \lambda} \Psi(t-\tau(t)) \\
& +d_{m} \dot{\Phi}^{T}(t) Z_{1 \lambda} \dot{\Phi}(t)-\frac{1}{d_{m}}(\Phi(t) \\
& \left.-\Phi\left(t-d_{m}\right)\right)^{T} Z_{1 \lambda}\left(\Phi(t)-\Phi\left(t-d_{m}\right)\right) \\
& +\left(d_{M}-d_{m}\right) \dot{\Phi}^{T}(t) Z_{2 \lambda} \dot{\Phi}(t) \\
& -\frac{1}{d_{M}-d_{m}}\left(\Phi\left(t-d_{m}\right)-\Phi(t-d(t))\right)^{T} \\
& \times Z_{2 \lambda}\left(\Phi\left(t-d_{m}\right)-\Phi(t-d(t))\right) \\
& +\tau_{m} \dot{\Psi}^{T}(t) Z_{3 \lambda} \dot{\Psi}(t)-\frac{1}{\tau_{m}}\left(\Psi(t)-\Psi\left(t-\tau_{m}\right)\right)^{T} \\
& \times Z_{3 \lambda}\left(\Psi(t)-\Psi\left(t-\tau_{m}\right)\right) \\
& +\left(\tau_{M}-\tau_{m}\right) \dot{\Psi}^{T}(t) Z_{4 \lambda} \dot{\Psi}(t) \\
& -\frac{1}{\tau_{M}-\tau_{m}}\left(\Psi\left(t-\tau_{m}\right)-\Psi(t-\tau(t))\right)^{T} \\
& \times Z_{4 \lambda}\left(\Psi\left(t-\tau_{m}\right)-\Psi(t-\tau(t))\right) \\
& -2 h^{T}(\Psi(t-\tau(t))) R_{\lambda} h(\Psi(t-\tau(t))) \\
& +2 h^{T}(\Psi(t-\tau(t))) R_{\lambda} \Lambda \Psi(t-\tau(t)) \\
& +2 \zeta^{T}(t) X\left[A_{\lambda} \Phi(t)\right.
\end{aligned}
$$

$$
\begin{aligned}
& \left.+B_{\lambda} h(\Psi(t-\tau(t)))-\dot{\Phi}(t)\right] \\
& +2 \zeta^{T}(t) Y\left[C_{\lambda} \Psi(t)+D_{\lambda} \Phi(t-d(t))-\dot{\Psi}(t)\right] \\
= & \zeta^{T}(t)\left(\Xi_{\lambda}+\Pi_{\lambda}+\Pi_{\lambda}^{T}\right) \zeta(t)
\end{aligned}
$$

where $\Xi_{\lambda}+\Pi_{\lambda}+\Pi_{\lambda}^{T}=\Xi_{i}+\Pi_{i}+\left.\Pi_{i}^{T}\right|_{i=\lambda}$ (all the subscripts $i$ in $\Xi_{i}+\Pi_{i}+\Pi_{i}^{T}$ are replaced with $\left.\lambda\right)$ and $\Xi_{i}, \Pi_{i}$ are given in (14).

On the other hand, for any $\lambda$ satisfying $\sum_{i=1}^{s} \lambda_{i}=1, \lambda_{i} \geq 0$, we have

$$
\sum_{i=1}^{s} \lambda_{i}\left(\Xi_{i}+\Pi_{i}+\Pi_{i}^{T}\right)=\Xi_{\lambda}+\Pi_{\lambda}+\Pi_{\lambda}^{T}<0 .
$$

Therefore, from (20) we have $\dot{V}(t)<0$ for all nonzero $\zeta(t)$. This means that the genetic regulatory network in (11) is robustly asymptotically stable for all admissible parameters $\left(A_{\lambda}, B_{\lambda}, C_{\lambda}, D_{\lambda}\right) \in \Gamma[18]$.

It is worth noting that in deriving the robust stability condition in Theorem 1, some novel ideas have been introduced to reduce the conservativeness.

1. Firstly, a new Lyapunov-Krasovskii functional has been introduced. The advantages of this new LyapunovKrasovskii functional are two folds. On one hand, it deals with the time-varying delay $d(t)$ (with both lower bound $d_{m}$ and upper bound $d_{M}$ ) as two successive delay components, that is, $d_{m}$ and $d(t)-d_{m}$, and the corresponding Lyapunov-Krasovskii functional in (15) aims at treating these two delay components separately. In such a way, both the lower bound $d_{m}$ and upper bound $d_{M}$ has been brought into the final robust stability condition. On the other hand, the Lyapunov-Krasovskii functional in (15) is dependent on the uncertain parameter $\lambda$, which is in contrast to the well-established robust stability notion in the quadratic framework that uses a fixed Lyapunov function for the entire uncertainty domain.

2. In deriving the robust stability in Theorem 1, no model transformation has been performed. It is noted that in deriving delay-dependent stability conditions, a common approach is to transform the original system to another one by using the Newton-Leibniz formula. In this framework, usually we have to employ some bounding techniques to find upper bounds for the inner product between two vectors. These bounding techniques involve some matrix inequalities. Employing these inequalities will inevitably increase conservatism into the derived conditions. However, it is worth emphasizing that in our derivation, no system transformation and thus no inequality is needed for seeking upper bounds of the inner product between two vectors (see the Proof of Theorem 1). This feature has the potential to enable us to obtain less-conservative results.

For Case II, we have the following theorem.

Theorem 2: The genetic network in (11) with time varying delays satisfying Case II is robustly asymptotically stable if there exist matrices $P_{i} \triangleq\left[\begin{array}{ll}P_{1 i} & P_{2 i} \\ P_{2 i}^{T} & P_{3 i}\end{array}\right]>0, Q_{j i} \geq 0, Z_{j i}>0$, $i=1, \ldots, s, j=1,3$, , and diagonal matrices $R_{i}, i=1, \ldots, s$, satisfying

$$
\bar{\Xi}_{i}+\Pi_{i}+\Pi_{i}^{T}<0, \quad i=1, \ldots, s
$$

where $\bar{\Xi}_{i}=\left.\Xi_{i}\right|_{Q_{2 i}=Q_{4 i}=0}$, and $\Pi_{i}$ is given in (14). 


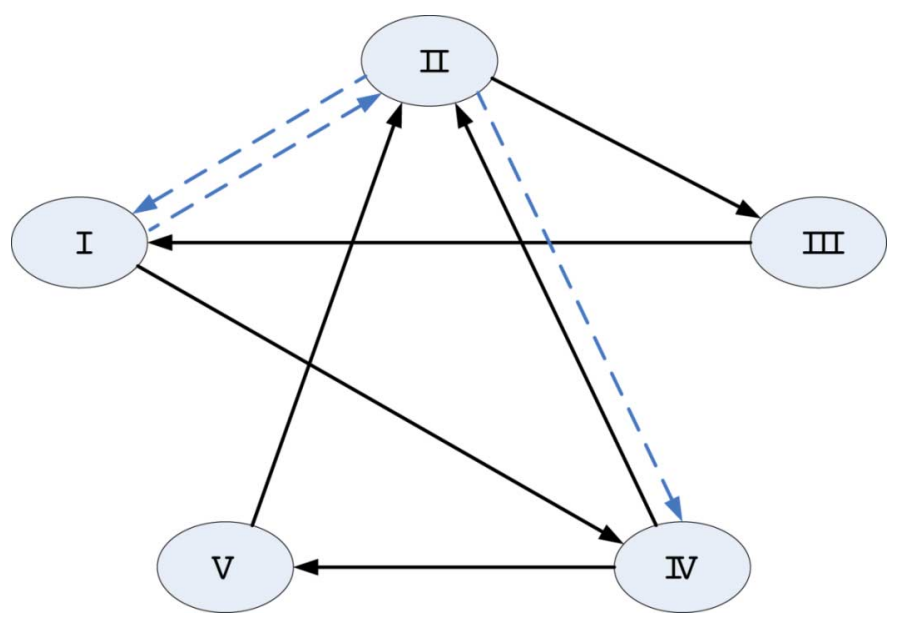

Fig. 1. A genetic network model.

Proof: Consider the following Lyapunov-Krasovskii functional:

$$
\begin{aligned}
V(t)= & V_{1}(t)+\bar{V}_{2}(t)+V_{3}(t) \\
\bar{V}_{2}(t)= & \int_{t-d_{m}}^{t} \Phi^{T}(s) Q_{1 \lambda} \Phi(s) d s \\
& +\int_{t-\tau_{m}}^{t} \Psi^{T}(s) Q_{3 \lambda} \Psi(s) d s
\end{aligned}
$$

where $V_{1}(t)$ and $V_{3}(t)$ are given in (15). Then, the theorem can be proved along similar lines as in the proof of Theorem 1.

Remark 5: The robust stability conditions given in both Theorem 1 and Theorem 2 are linear matrix inequalities over the decision variables to be determined. These conditions can be easily verified using some standard numerical software.

\section{ILLUSTRATIVE EXAMPLE}

In this section, we use two examples to show the usefulness of our theoretical results developed. The first example is a numerical one on a genetic network with five nodes, and the second example is concerned with a synthetic oscillatory network of transcriptional regulators with three repressor-protein concentrations and their corresponding mRNA concentrations as the continuous dynamical variables.

Example 1: Consider a small size (but of complex coupling topology) genetic network with five nodes, shown in Fig. 1. In Fig. 1, each ellipse represents a gene, and the lines between two genes represent regulatory links, in which the solid line and dashed line denote activation and repression respectively. It is assumed that the dimensionless transcriptional rates are all 0.5. According to the definition of links in Section II, we can obtain the coupling matrix $B$ of this network as

$$
B=0.5 \times\left[\begin{array}{ccccc}
0 & -1 & 1 & 0 & 0 \\
-1 & 0 & 0 & 1 & 1 \\
0 & 1 & 0 & 0 & 0 \\
1 & -1 & 0 & 0 & 0 \\
0 & 0 & 0 & 1 & 0
\end{array}\right]
$$

and $\rho=0.5 \times\left[\begin{array}{lllll}1 & 1 & 0 & 1 & 0\end{array}\right]$ in (7). We consider the genetic network in Fig. 1 with time-varying delays. Let the system matrices in (11) be given as follows:

$$
\begin{aligned}
& A_{\lambda}=C_{\lambda}=\left(1+\delta_{1}\right) \operatorname{diag}\{-1,-1,-1,-1,-1\}, \\
& B_{\lambda}=\left(1+\delta_{2}\right) B, \\
& D_{\lambda}=\operatorname{diag}\{1,1,1,1,1\},
\end{aligned}
$$

where $\left|\delta_{1}\right| \leq 0.1$ and $\left|\delta_{2}\right| \leq 0.2$ represent uncertain parameters. Then, the above uncertain genetic network can be represented by a four-vertex polytopic genetic network, with four vertex matrices given by

$$
\begin{aligned}
& A_{1}=C_{1}=\operatorname{diag}\{-1.1,-1.1,-1.1,-1.1,-1.1\}, \\
& B_{1}=0.8 B, \quad D_{1}=\operatorname{diag}\{1,1,1,1,1\} ; \\
& A_{2}=C_{2}=\operatorname{diag}\{-0.9,-0.9,-0.9,-0.9,-0.9\}, \\
& B_{2}=0.8 B, \quad D_{2}=\operatorname{diag}\{1,1,1,1,1\} ; \\
& A_{3}=C_{3}=\operatorname{diag}\{-1.1,-1.1,-1.1,-1.1,-1.1\}, \\
& B_{3}=1.2 B, \quad D_{3}=\operatorname{diag}\{1,1,1,1,1\} ; \\
& A_{4}=C_{4}=\operatorname{diag}\{-0.9,-0.9,-0.9,-0.9,-0.9\}, \\
& B_{4}=1.2 B, \quad D_{4}=\operatorname{diag}\{1,1,1,1,1\} .
\end{aligned}
$$

In addition, let the function $f_{i}\left(x_{i}\right)$ in (7) be given by

$$
f_{i}\left(x_{i}\right)=\frac{x_{i}^{2}}{1+x_{i}^{2}},
$$

i.e., the Hill coefficient is 2 . It is easy to know that the maximal value of the derivative of $f_{i}\left(x_{i}\right)$ is $a_{i}=0.65$. The time delays are assumed to be

$$
d(t)=(1+0.2 \sin 5 t) / 4, \quad \tau(t)=(1+0.2 \cos 4 t) / 4
$$

and therefore we can get the parameters as follows:

$$
\begin{array}{rll}
d_{M}=0.3, & d_{m}=0.2, & \tau_{M}=0.3, \\
\tau_{m}=0.2, & \varepsilon_{d}=0.25, & \varepsilon_{\tau}=0.2 .
\end{array}
$$

By solving the condition in Theorem 1 using the LMI toolbox in Matlab, we can obtain a feasible solution with the following obtained matrix variables (for space consideration, here we only list the matrix variables $P_{1 i}$ and $R_{i}$, at the bottom of the next page): This shows that this genetic network is robustly asymptotically stable.

Example 2.: In this example, we use a synthetic oscillatory network of transcriptional regulators to further illustrate our result. This network has been adopted as the mathematical model of the repressilator, which has been experimentally investigated in [9]. In this model, three repressor-protein concentrations, $p_{i}$, and their corresponding mRNA concentrations, $m_{i}$ (where $i$ is lacl, tet $R$ or $c l$ ) are treated as continuous dynamical variables. Each of these six molecular species participates in transcription, translation, and degradation reactions. Here we consider only the symmetrical case in which all three repressors are 
identical except for their DNA-binding specificities. By incorporating time delays, the kinetics of the system are determined by six coupled first-order differential equations:

$$
\begin{aligned}
\dot{m}_{i}(t) & =-m_{i}(t)+\frac{\alpha}{1+p_{k}^{n}(t-\tau(t))}+\alpha_{0}, \\
\dot{p}_{i}(t) & =-\beta p_{i}(t)+\beta m_{i}(t-d(t))
\end{aligned}
$$

where $i$ and $k$ have the following three pairs of values: $(i=1$, $k=2),(i=2, k=3),(i=3, k=1) . n$ is the Hill coefficient. $d(t)$ and $\tau(t)$ are the delay times for the proteins and mRNA, respectively. From (22) we have

$$
\begin{aligned}
\dot{m}_{i}(t) & =-m_{i}(t)+(-\alpha) \frac{p_{k}^{n}(t-\tau(t))}{1+p_{k}^{n}(t-\tau(t))}+\alpha+\alpha_{0}, \\
\dot{p}_{i}(t) & =-\beta p_{i}(t)+\beta m_{i}(t-d(t)) .
\end{aligned}
$$

Then, by selecting

$$
\begin{aligned}
\bar{\phi}(t) & =\left[\begin{array}{l}
m_{1}(t) \\
m_{2}(t) \\
m_{3}(t)
\end{array}\right], \quad \bar{\psi}(t)=\left[\begin{array}{l}
p_{1}(t) \\
p_{2}(t) \\
p_{3}(t)
\end{array}\right], \\
\rho & =\left[\begin{array}{l}
a+\alpha_{0} \\
a+\alpha_{0} \\
a+\alpha_{0}
\end{array}\right], \\
\bar{\phi}(t-d(t))) & =\left[\begin{array}{l}
m_{1}(t-d(t)) \\
m_{2}(t-d(t)) \\
m_{3}(t-d(t))
\end{array}\right], \\
f(\bar{\psi}(t-\tau(t))) & =\left[\begin{array}{l}
f_{1}\left(p_{1}(t-\tau(t))\right) \\
f_{2}\left(p_{2}(t-\tau(t))\right) \\
f_{n}\left(p_{3}(t-\tau(t))\right)
\end{array}\right]
\end{aligned}
$$

we obtain system (7) with the following parameters:

$$
\begin{aligned}
A & =\operatorname{diag}\{-1,-1,-1\}, \quad C=\operatorname{diag}\{-\beta,-\beta,-\beta\}, \\
D & =\operatorname{diag}\{\beta, \beta, \beta\}, \\
B & =\left[\begin{array}{ccc}
0 & -\alpha & \alpha \\
\alpha & 0 & -\alpha \\
-\alpha & \alpha & 0
\end{array}\right] .
\end{aligned}
$$

Let the Hill coefficient be 2. Then we have $a_{i}=0.65$ for (10). In addition, assume the parameters $\alpha=0.5$ and $\beta=1$. In this example, we assume the time delays to be

$$
\begin{aligned}
d_{M} & =0.3 \mathrm{~s}, \quad d_{m}=0.2999 \mathrm{~s}, \quad \tau_{M}=1 \mathrm{~s}, \\
\tau_{m} & =0.9999 \mathrm{~s}, \quad \varepsilon_{d}=0, \quad \varepsilon_{\tau}=0 .
\end{aligned}
$$

By solving the condition in Theorem 1 using the LMI toolbox in Matlab, we can obtain a feasible solution with the following obtained matrix variables (for space consideration, here we only list the matrix variables $P_{1}, P_{2}, P_{3}$ and $R$ ):

$$
\begin{aligned}
P_{1} & =\left[\begin{array}{ccc}
12.6008 & 0.2433 & 0.2433 \\
0.2433 & 12.6008 & 0.2433 \\
0.2433 & 0.2433 & 12.6008
\end{array}\right], \\
P_{2} & =\left[\begin{array}{ccc}
-0.2871 & 0.1839 & 0.1912 \\
0.1912 & -0.2871 & 0.1839 \\
0.1839 & 0.1912 & -0.2871
\end{array}\right], \\
P_{3} & =\left[\begin{array}{ccc}
11.2592 & 0.2772 & 0.2772 \\
0.2772 & 11.2592 & 0.2772 \\
0.2772 & 0.2772 & 11.2592
\end{array}\right], \\
R & =\operatorname{diag}\{8.1294,8.1294,8.1294\} .
\end{aligned}
$$

$$
\begin{aligned}
& P_{11}=\left[\begin{array}{ccccc}
0.0421 & -0.0002 & 0.0026 & -0.0030 & -0.0005 \\
-0.0002 & 0.0394 & -0.0009 & 0.0031 & -0.0014 \\
0.0026 & -0.0009 & 0.0425 & 0.0025 & 0.0001 \\
-0.0030 & 0.0031 & 0.0025 & 0.0403 & 0.0012 \\
-0.0005 & -0.0014 & 0.0001 & 0.0012 & 0.0436
\end{array}\right], \\
& P_{12}= {\left[\begin{array}{ccccc}
0.0414 & -0.0006 & 0.0036 & -0.0042 & -0.0007 \\
-0.0006 & 0.0376 & -0.0012 & 0.0041 & -0.0022 \\
0.0036 & -0.0012 & 0.0419 & 0.0034 & 0.0001 \\
-0.0042 & 0.0041 & 0.0034 & 0.0391 & 0.0016 \\
-0.0007 & -0.0022 & 0.0001 & 0.0016 & 0.0434
\end{array}\right], } \\
& P_{13}= {\left[\begin{array}{ccccc}
0.0422 & -0.0000 & 0.0021 & -0.0026 & -0.0004 \\
-0.0000 & 0.0398 & -0.0008 & 0.0026 & -0.0012 \\
0.0021 & -0.0008 & 0.0425 & 0.0022 & 0.0001 \\
-0.0026 & 0.0026 & 0.0022 & 0.0405 & 0.0009 \\
-0.0004 & -0.0012 & 0.0001 & 0.0009 & 0.0435
\end{array}\right], } \\
& P_{14}= {\left[\begin{array}{ccccc}
0.0415 & -0.0001 & 0.0030 & -0.0036 & -0.0005 \\
-0.0001 & 0.0381 & -0.0011 & 0.0036 & -0.0020 \\
0.0030 & -0.0011 & 0.0420 & 0.0030 & 0.0001 \\
-0.0036 & 0.0036 & 0.0030 & 0.0393 & 0.0012 \\
-0.0005 & -0.0020 & 0.0001 & 0.0012 & 0.0434
\end{array}\right], } \\
& R_{1}=\operatorname{diag}\{0.0219,0.0219,0.0219,0.0219,0.0219\}, \\
& R_{2}=\operatorname{diag}\{0.0336,0.0336,0.0336,0.0336,0.0336\}, \\
& R_{3}=\operatorname{diag}\{0.0214,0.0214,0.0214,0.0214,0.0214\}, \\
& R_{4}=\operatorname{diag}\{0.0288,0.0288,0.0288,0.0288,0.0288\}
\end{aligned}
$$


This shows that the synthetic oscillatory network of transcriptional regulators is asymptotically stable under the above conditions.

\section{CONCLUSION}

This paper has presented two robust asymptotic stability conditions for genetic regulatory networks with differentiable or nondifferentiable time-varying delays. Convex polytopic description is utilized to characterize the genetic network model uncertainties. These conditions are formulated in terms of linear matrix inequalities, which can be readily solved by using standard numerical software. By utilizing some advanced techniques, both the upper and lower bounds of the delays are brought into the final robust stability conditions. Another feature of the results lies in that a novel Lyapunov functional dependent on the uncertain parameters has been utilized, which leads the results to be potentially less conservative than those obtained by using a fixed Lyapunov functional for the entire uncertainty domain. A genetic network example has illustrated the applicability and usefulness of the developed theoretical results.

\section{REFERENCES}

[1] A. Becskel and L. Serrano, "Engineering stability in gene networks by autoregulation," Nature, vol. 405, no. 6786, pp. 590-593, 2000.

[2] F. A. Bignone, R. Livi, and M. Propato, "Dynamical stability and finite amplitude perturbations in coupled genetic networks," Physica D: Nonlinear Phenom., vol. 108, no. 4, pp. 379-396, 1997.

[3] H. Bolouri and E. H. Davidson, "Modeling transcriptional regulatory networks," BioEssays, vol. 24, no. 12, pp. 1118-1129, 2002.

[4] E. K. Boukas and Z.-K. Liu, "Deterministic and stochastic time-delay systems," in Birkhauser, Boston, 2002.

[5] S. Boyd, L. El Ghaoui, E. Feron, and V. Balakrishnan, "Linear matrix inequalities in systems and control theory," in SIAM, Philadelphia, PA, 1994.

[6] L. Chen and K. Aihara, "Stability and bifurcation analysis of differential-difference-algebraic equations," IEEE Trans. Circuits Syst. I, Fundam. Theory Appl., vol. 48, no. 3, pp. 308-326, Mar. 2001.

[7] L. Chen and K. Aihara, "Stability of genetic regulatory networks with time delay," IEEE Trans. Circuits Syst. I, Fundam. Theory Appl., vol. 49, no. 5, pp. 602-608, May 2002.

[8] H. De Jong, "Modeling and simulation of genetic regulatory systems: A literature review," J. Comput. Biol., vol. 9, no. 1, pp. 67-103, 2002.

[9] M. B. Elowitz and S. Leibler, "A synthetic oscillatory network of transcriptional regulators," Nature, vol. 403, no. 20, pp. 335-338, 2000.

[10] Y. Fukuta, Y. A. Chapuis, Y. Mita, and H. Fujita, "Design, fabrication, and control of MEMS-based actuator arrays for air-flow distributed micromanipulation," J. Microelectromech. Syst., vol. 15, pp. 912-926, 2006.

[11] P. Gahinet, A. Nemirovskii, A. J. Laub, and M. Chilali, LMI Control Toolbox User's Guide. Natick, MA: The Math Works Inc., 1995.

[12] H. Gao and C. Wang, "Delay-dependent robust $H_{\infty}$ and $L_{2}-L_{\infty}$ filtering for a class of uncertain nonlinear time-delayed systems," IEEE Trans. Autom. Control, vol. 48, no. 9, pp. 1661-1666, Sep. 2003.

[13] H. Gao and C. Wang, "A delay-dependent approach to robust $H_{\infty}$ filtering for uncertain discrete-time state-delayed systems," IEEE Trans. Signal Process., vol. 52, no. 6, pp. 1631-1640, Jun. 2004.

[14] H. Gao and T. Chen, "New results on stability of discrete-time systems with time-varying state delay," IEEE Trans. Autom. Control, vol. 52, no. 2, pp. 328-334, Feb. 2007.

[15] T. S. Gardner, C. R. Cantor, and J. J. Collins, "Construction of a genetic toggle switch in escherichia coli," Nature, vol. 403, no. 20, pp. $339-342,2000$.

[16] B. Grammaticos, A. S. Carstea, and A. Ramani, "On the dynamics of a gene regulatory network," J. Phys. A, Math. Gen., vol. 39, no. 12, pp. 2965-2971, 2006.

[17] K. Gu, V. L. Kharitonov, and J. Chen, Stability of Time-Delay Systems.. Berlin, Germany: Springer-Verlag, 2003.

[18] J. Hale and S. M. V. Lunel, Introduction to Functional Differential Equations. Berlin, Germany: Springer, 1993.
[19] Y. He, Q. G. Wang, C. Lin, and M. Wu, "Delay-range-dependent stability for systems with time-varying delay," Automatica, vol. 43, no. 2, pp. 371-376, 2007.

[20] S. Kalir, S. Mangan, and U. Alon, "A coherent feed-forward loop with a sum input function prolongs flagella expression in escherichia coli," Mol. Syst. Biol. [Electronic Resource], vol. 1, 2005, doi:10.1038/msb4100010.

[21] T. Kobayashi, L. Chen, and K. Aihara, "Modeling genetic switches with positive feedback loops," J. Theor. Biol., vol. 221, no. 3, pp. 379-399, 2003.

[22] C. Li, L. Chen, and K. Aihara, "Stability of genetic networks with sum regulatory logic: Lur'e system and lmi approach," IEEE Trans. Circuits Syst. I, Reg.Papers, vol. 53, no. 11, pp. 2451-2458, Nov. 2006.

[23] C. Lin, Q. G. Wang, and T. H. Lee, "A less conservative robust stability test for linear uncertain time-delay systems," IEEE Trans. Autom. Control, vol. 51, no. 1, pp. 87-91, Jan. 2006.

[24] J. M. Mahaffy and C. V. Pao, "Models of genetic control by repression with time delays and spatial effects," J. Math. Biol., vol. 20, pp. 39-57, 1984.

[25] M. Malek-Zavarei and M. Jamshidi, Time-Delay Systems: Analysis, Optimation and Application. Amsterdam, The Netherlands: NorthHolland, 1987.

[26] H. H. McAdams and L. Shapiro, "Circuit simulation of genetic networks," Science, vol. 269, no. 5224, pp. 650-656, 1995.

[27] N. A. M. Monk, "Oscillatory expression of hes1, p53, and NF- $\kappa$ B driven by transcriptional time delays," Current Biol., vol. 13, pp. 1409-1413, 2003.

[28] Y. S. Moon, P. G. Park, W. H. Kwon, and Y. S. Lee, "Delay-dependent robust stabilization of uncertain state-delayed systems," Int. J. Control, vol. 74, pp. 1447-1455, 2001.

[29] M. Pizza, V. Scarlato, and V. Masignani, "Identification of vaccine candidates against serogroup $\mathrm{b}$ meningococcus by whole-genome sequencing," Science, vol. 287, pp. 1816-1820, 2000.

[30] F. Ren and J. Cao, "Asymptotic and robust stability of genetic regulatory networks with time-varying delays," Neurocomputing, vol. 71, no. 4-6, pp. 834-842, 2008.

[31] P. Smolen, D. A. Baxter, and J. H. Byrne, "Mathematical modeling of gene networks," Neuron, vol. 26, no. 26, pp. 567-580, 2000.

[32] L. L. Tu and J. A. Lu, "Stability of a model for a delayed genetic regulatory network," Dyn. Continuous, Discr. Impulsive Syst. Series B, Appl. Algorithms, vol. 13, no. 3-4, pp. 429-439, 2006.

[33] Z. Wang, H. Shu, J. Fang, and X. Liu, "Robust stability for stochastic Hopfield neural networks with time delays," Nonlinear Anal., Real World Appl., vol. 7, no. 5, pp. 1119-1128, 2006.

[34] Z. Wang, F. Yang, D. W. C. Ho, and X. Liu, "Robust $H_{\infty}$ filtering for stochastic time-delay systems with missing measurements," IEEE Trans. Signal Process., vol. 54, no. 7, pp. 2579-2587, Jul. 2006.

[35] Z. Wang, Y. Liu, M. Li, and X. Liu, "Stability analysis for stochastic Cohen-Grossberg neural networks with mixed time delays,", IEEE Trans. Neural Netw., vol. 17, no. 3, pp. 814-820, May 2006.

[36] B. Xu and Y. Tao, "External noise and feedback regulation: Steadystate statistics of auto-regulatory genetic network," J. Theor. Biol., vol. 243, no. 2, pp. 214-221, 2006.

[37] C. H. Yuh, H. Bolouri, and E. H. Davidson, "Genomic cis-regulatory logic: Experimental and computational analysis of a sea urchin gene," Science, vol. 279, no. 5358, pp. 1896-1902, 1998.

[38] K. Zhou, J. C. Doyle, and K. Glover, Robust and Optimal Control. Englewood Cliffs, NJ: Prentice-Hall, 1996.

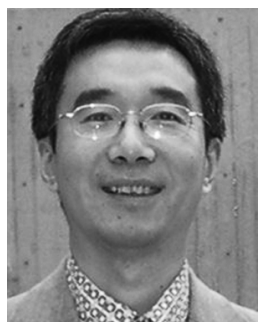

Zidong Wang (SM'03) was born in Jiangsu, China, in 1966. He received the B.Sc. degree in mathematics in 1986 from Suzhou University, Suzhou, China, and the M.Sc. degree in applied mathematics and the $\mathrm{Ph} . \mathrm{D}$. degree in electrical and computer engineering from Nanjing University of Science and Technology, Nanjing, China, in 1990 and 1994, respectively.

Dr. Wang is now a Professor of Dynamical Systems and Computing at Brunel University, U.K. He was appointed as Lecturer in 1990 and Associate Professor in 1994 at Nanjing University of Science and Technology. From January 1997 to December 1998, he was an Alexander von Humboldt research fellow with the Control Engineering Laboratory, Ruhr-University Bochum, Germany. From January 1999 to February 2001, he was a Lecturer with Department of Mathematics, University of Kaiserslautern, Germany. From March 2001 to July 2002, he was a University Senior Research Fellow 
with the School of Mathematical and Information Sciences, Coventry University, U.K. In August 2002, he joined the Department of Information Systems and Computing, Brunel University, U.K., as a Lecturer, and was promoted to Reader in September 2003 and to Professor in July 2007. He also holds an Adjunct TePin Professorship at Donghua University, Shanghai, China, a Visiting Professorship at Fuzhou University, Fuzhou, China, and a Guest Professorship at Nanjing Normal University, Nanjing, China. He has published more than 80 papers in refereed international journals. His research interests include dynamical systems, signal processing, bioinformatics, control theory and applications.

Dr. Wang was awarded the Humboldt research fellowship in 1996 from the Alexander von Humboldt Foundation, the JSPS Research Fellowship in 1998 from the Japan Society for the Promotion of Science, and the William Mong Visiting Research Fellowship in 2002 from the University of Hong Kong. He was a recipient of the Outstanding Science and Technology Development Awards, once in 2005 and twice in 1997 from the National Education Committee of China, once in 1997 from the Military Industry General Company of China, once in 1997 from Jiangsu Province Government of China, and once in 1999 from the National Education Ministry of China.

Dr. Wang currently serves as an Associate Editor for IEEE TRANSACTIONS on Automatic Control, IEEE Transactions on Neural Networks, IEEE TRansactions on Signal PROCESSING, IEEE TRANSACtions ON Systems, Man, and Cybernetics-PART C, IEEE TRANSACTIONS ON Control Systems TeChnology, Circuits, Systems \& Signal Processing, an Action Editor for Neural Networks, an Editorial Board Member for International Journal of Systems Science, Neurocomputing, International Journal of Computer Mathematics, International Journal of General Systems, and an Associate Editor on the Conference Editorial Board for the IEEE Control Systems Society. He is a Senior Member of the IEEE, a Fellow of the Royal Statistical Society, a member of program committee for many international conferences, and a very active reviewer for many international journals. $\mathrm{He}$ was nominated an appreciated reviewer for IEEE TRANSACTIONS ON SIGNAL PROCESSING in 2006, an outstanding reviewer for IEEE TRANSACTIONS ON Automatic Control in 2004 and for the journal Automatica in 2000. He received the Standing Membership of the Technical Committee on Control of IASTED (International Association of Science and Technology for Development) in 2000. From 2001 to 2007 he served as a member of technical program committee for 35 international conferences in the general area of systems theory and computing.

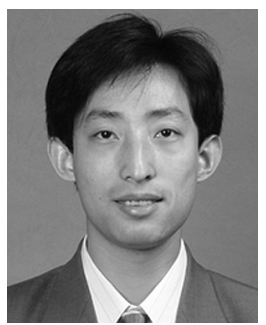

Huijun Gao (M'06) was born in Heilongjiang Province, China, in 1976. He received the M.S. degree in electrical engineering from Shenyang University of Technology, Shengyang, China, in 2001 and the Ph.D. degree in control science and engineering from Harbin Institute of Technology, Harbin, China, in 2005.

He was a Research Associate at the Department of Mechanical Engineering, University of Hong Kong, Hong Kong, from November 2003 to August 2004, and joined Harbin Institute of Technology in November 2004, where he is currently a Professor. He carried out his postdoctoral research in the Department of Electrical and Computer Engineering, University of Alberta, Edmonton, $\mathrm{AB}$, Canada, during his leave from Harbin Institute of Technology from October 2005 to October 2007. His research interests include network-based control, robust control/filter theory, model reduction, time-delay systems, and multidimensional systems, and their applications.
Dr. Gao is an Associate Editor or member of editorial board for several journals, such as IEEE TRANSACTIONS ON SYSTEMS, MAN, AND CYBERNETICS-PART B: CYBERnETICS, International Journal of Systems Science, Journal of Intelligent and Robotics Systems, and Journal of the Franklin Institute. He was the recipient of the University of Alberta Dorothy J. Killam Memorial Postdoctoral Fellow Prize in 2005 and corecipient of the Outstanding Science and Technology Development Awards from the Ministry of Machine-Building Industry of China and from the Liaoning Provincial Government of China, both in 2002. In 2005, he was awarded an Alberta Ingenuity Fellowship and a University of Alberta Honorary Izaak Walton Killam Memorial Postdoctoral Fellowship. He was an Outstanding Reviewer for Automatica in 2007 and an Appreciated Reviewer for the IEEE TRANSACTIONS ON SigNAL PROCESSING in 2006. He received the National Excellent Doctoral Dissertation Award in 2007 from the Ministry of Education of China.

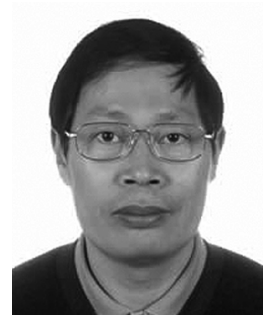

Jinde Cao (M07-SM07) received the B.S. degree in mathematics/applied mathematics from Anhui Normal University, Wuhu, China, in 1986, the M.S. degree in mathematics/applied mathematics from Yunnan University, Kunming, China, in 1989, and the $\mathrm{Ph} . \mathrm{D}$. degree in mathematics/applied mathematics from Sichuan University, Chengdu, China, in 1998

From March 1989 to May 2000, he was with Yunnan University. In May 2000, he joined the Department of Mathematics, Southeast University, Nanjing, China. From July 2001 to June 2002, he was a Postdoctoral Research Fellow with the Department of Automation and Computer-aided Engineering, Chinese University of Hong Kong, Kowloon, Hong Kong. In 2006 and 2007, he was a Visiting Research Fellow and a Visiting Professor with the School of Information Systems, Computing and Mathematics, Brunel University, London, U.K. He is currently a TePin Professor and Doctoral Advisor with Southeast University, prior to which, he was a Professor with Yunnan University from 1996 to 2000 . He is the author or coauthor of more than 150 journal papers and five edited books and a Reviewer of mathematical reviews and Zentralblatt-Math. His research interests include nonlinear systems, neural networks, complex systems and complex networks, stability theory, and applied mathematics.

Dr. Cao an Associate Editor of the IEEE TRAnsactions on NeuraL NETWORKS, Journal of the Franklin Institute, Mathematics and Computers in Simulation, and Neurocomputing.

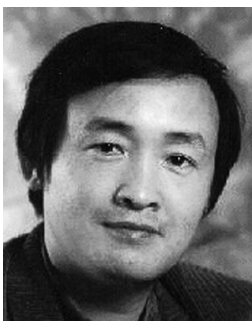

Xiaohui Liu is a Professor of Computing at Brunel University, U.K. He leads the Intelligent Data Analysis (IDA) Group, performing interdisciplinary research involving artificial intelligence, dynamic systems, image and signal processing, and statistics, particularly for applications in biology, engineering, and medicine. He serves on editorial boards of four computing journals, founded the biennial international conference series on IDA in 1995, and has given numerous invited talks in bioinformatics, data mining, and statistics conferences. 